Inorg. Chim. Acta. 2004.

\title{
Control mechanisms for transport and nonlinear optical response in organic materials: A tale of twists and barriers.
}

\author{
Antonio Facchetti, Geoffrey R. Hutchison, Shahar Keinan and Mark A. Ratner* \\ Department of Chemistry and the Materials Research Center, Northwestern University, \\ 2145 Sheridan Road, Evanston, Illinois, 60208-3113. \\ * To whom correspondence should be addressed: \\ $\underline{\text { ratner@chem.northwestern.edu }}$
}




\begin{abstract}
Simple electronic structure models are used to address two significant challenges in organic materials chemistry, the design of chromophores for strong electro-optic response (and low-energy optical absorption), and the prediction of relative mobilities and charge injection barriers for conductive oligomers.

For electro-optic response, we examine two chromophore classes where twisting around an inter-ring bond can tune the electronic structure from aromatic (zwitterionic) to quinoid (neutral). The calculated nonlinear response develops a very strong maximum $\left(\beta_{\mu} \sim 1500^{*} 10^{-30}\right.$ esu) at twist angles near $80^{\circ}$. For the transport behavior, structure/function correlations are presented for three series of oligomers, based on calculations of bandwidths (as functions of geometry) and of reorganization energies. Transport type appears to be fixed less by these mobility factors than by the injection barriers. The simplest estimates for these Schottky-type barriers, using frontier orbital energies from density functional calculations, predict carrier n-type or p-type behavior remarkably well.
\end{abstract}




\section{Introduction}

Smart, efficient design and fabrication of organic-based electronics and electrooptics are enabled by three major trends. The structural, electronic, and physical properties of organic molecules, oligomers and polymers can be broadly modified ("tuned") by chemical substituents and heterocyclic patterns. Synthesis of such modified structures is becoming progressively easier, due largely to newly developed organometallic-based coupling chemistry. [1] Computational methods for calculating structural, electronic and response properties of organics have developed to the point that many properties can be predicted quantitatively. Based on these advances, organic-based electronics and electro-optics are now studied in a very broad area of science and technology.

In this article, we follow the lead of Tobin Marks, and investigate mechanisms for control of particular aspects of organic electronics. Section II is devoted to nonlinear optics, in particular to further investigation of a class of molecules whose response is governed by an internal bond rotation, or twist. These species were suggested a few years ago by the Marks' group; here we extend that original analysis both by using a more sophisticated (and more reliable) multi-configurational method, and by broadening the considerations to a new chromophore set, that permits self-conjugation, to form selfassembled superlattices, while retaining the ability to tune the NLO response with twisting.

Section III is devoted to an analysis of charge mobility (both for hole and for electron) in a series of functionalized heterocyclic oligomers. At ambient temperature, the narrow bands and strong vibronic coupling that are typical for organic solids, result in 
activated, hopping-type transport. The mobility is then proportional to the nearest neighbor hopping rate. Using the Marcus picture, this rate is proportional to the square of the Hamiltonian matrix element between nearest neighbor sites, and to $\exp \{-\lambda / 4 \mathrm{kT}\}$, where $\lambda$ is the total reorganization energy for a hole or electron hopping process. We calculate both $\lambda$ and the matrix elements, and use the results to deduce relative mobilities for different species, and for different geometries. The optimum transport geometry is fixed by the matrix elements, but the variation in mobility from one species to another generally arises from differing reorganization energies.

In section IV we use a greatly simplified Schottky-barrier model to compute (from the frontier orbital energy values) relative rates for hole or electron injection. Section V contains some final remarks.

\section{A twisted tale of non-linear optics chromophores}

The realization of large-response electro-optic devices has been the focus of active research in the last decade [2], [3] to which the Marks group has contributed enormously. [4] Of special interest are materials composed of organic molecules with designed individual responses, [4e], [5] which undergo self-assembly to form a SAS (Self Assembled Superlattice) during device fabrication, in such a way as to avoid centrosymmetry [6] [7]. The resulting devices generally offer improved electro-optic properties such as fast response, low dielectric constant and tunability, compared to conventional inorganic materials. Such devices have applications in various photonic technologies, such as electro-optic switching for telecommunications, RF sensing, and

optical information processing. [8] Organic molecules exhibiting large electro-optic 
response are often composed of $\pi$-conjugated systems linking electron donor and acceptor substituents. The electronic structures of such molecules are highly sensitive to the nature of the donor and acceptor, as well as to any other structural modifications within the intervening $\pi$ system $[9],[10]$.

Albert et al. [9] offered a new approach to molecular chromophores that exhibit both large NLO responses and infrared-shifted absorption. Albert et al. [9] recognized a group of molecules where tuning the twist angle about a double bond allows control of the electronic structure and tuning of the NLO properties. The merocyanine-based dyes of Chart 1 are such molecules. Derivatives of those chromophores have two dominant electronic structure components, referred to as the "quinoid", IB, and "charge-separated", IA, forms. When the twist angle, $\phi$, is zero, the $\pi$ orbitals in the phenyl and pyridine rings are coplanar so that delocalization between the rings is strongest, leading to the quinoid state, IB. However, as the twist angle increases the intervening $\pi$-delocalization is diminished. When the twist angle is $90^{\circ}$, there is no inter-ring conjugation - this is the fully charge-separated state, IA.

Insert Chart 1 here.

The original report by Albert et al. [9] was followed by both experimental [11], [12] and theoretical [13] work. The theoretical work, however, employed simple and pragmatic CIS (Configuration Interaction Singles) methods based on an assumed HartreeFock ground state. However, a multi-reference representation (MRD/CI) [14] of the ground state generally gives far more accurate [15], [16] response properties than simple 
CIS, because the ground state (as well as the lowest lying excited state, which contributes most strongly to the overall $\beta$ in a state description) is often poorly represented by the SCF determinant and singly excited configurations, respectively. While some qualitative aspects of the calculation using simple CIS resemble those of the more accurate multireference calculation, the actual numerical values differ substantially, and the latter picture offers more insight into the electronic structures of these chromophores [15]. It was shown that the largest $\beta_{n}$ occurs near a twist angle of $80^{\circ}$, and is four times greater in the MRD/CI description than in the original CIS, so that the previous study [9], which suggested very large magnitudes of $\beta_{n}$ (see definition in Appendix A), actually underestimated them. Table 1 reproduces [15] both the computed first allowed excitation energy (in $\mathrm{eV}$ ) and the static $\beta_{n}$ as functions of the constrained dihedral twist angle $\phi$ about the central double bond for the MRD-CI calculations. A more detailed description of the calculation can be found in Appendix A and ref. [15].

Insert Table 1 and Figure 1 Here.

Changing the dihedral angle alters the electronic structure of the chromophore, as indicated by excitation energies and NLO response. In Figure 1 we plot the excitation energy to the lowest-lying excited state (left side) and $-\beta_{n}$ (right side) as a function of the twist angle. The excitation energy shifts to the red with increasing dihedral angle, in a gradual and monotonic fashion, indicating linear evolution from the quinoidal to chargeseparated form, with most chromophore structures in the set having dual character. The 
decrease in excitation energy with increasing dihedral angle is what one would expect in a two-site donor-acceptor Hückel model. [9], [17]

Accompanying the changes in the molecular optical excitation energy are the changes in the molecular hyperpolarizability. Interestingly, for large dihedral twist angles, the chromophores display negative hyperpolarizability, with the ground state dipole moment larger than the excited state dipole moment. For larger dihedral twist angles, the ground state is dominated by the zwitterionic charge separated form, while the first excited state will be the quinoid one [18], with a smaller dipole moments, thus giving rise to negative hyperpolarizability. The chromophore hyperpolarizability increases with the dihedral angle, maximizing at $\phi=80^{\circ}$. For dihedral angles near $90^{\circ}, \beta_{n}$ decreases because there is almost no conjugation between the phenyl and pyridyl $\pi$ systems.

Insert Chart 2 here.

Chromophore I represents a proof-of-concept. However, incorporation of this system into a multi-layer self-assembly scheme (for acentric film fabrication) is extremely difficult, since to the phenolate oxygen atom cannot be functionalized without destroying the quinoidal character of the chromophore and lowering the non-linear electrooptical response dramatically. Chart 2 shows the design of a new chromophore set that will keep the dual quinoidal charge separated character while permitting a SAS scheme. Figure 2 shows the schematic of the protection - deprotection SAS generation method developed and perfected in the Marks group. [4a], [5], [19] In this technique, the 
chromophore units in the SAS acentric face are bonded to the surface and among themselves via siloxane linkage. In chromophore set II an amide linker replaces the phenolate oxygen atom of chromophore set I, without dramatically altering the donoracceptor character of the original chromophore. In fact, using various $X_{1}, X_{2}, Y_{1}$ and $Y_{2}$ alkyl substituents, (see Table 2) it is possible to tune the dihedral twist angle and thus altering the linear- and non-linear optical response properties, as is shown in Table 2 and Figure 3. Chromophore set II shows the same trends in the optical and NLO response properties as chromophore set $\mathbf{I}$, including both gradual red shifts of the excitation energies with increasing twist angles and a molecular hyperpolarizability for large dihedral angles that is negative and peaked around twist angle of $80^{\circ}$. The amide linker changes donor polarity, improving slightly the electro-optic response properties in comparison to chromophore set $\mathbf{I}$.

Insert Figure $2 \& 3$ here.

Increasing the dihedral angle alters the electronic structure of the chromophore by lowering the conjugation between the phenyl and pyridine rings of the chromophores of set II. Figure 4 displays the HOMO and LUMO orbitals (from the singlet RHF INDO/S calculation) for the chromophore with $\phi=35^{\circ}$ (II-1), $\phi=60^{\circ}$, (II-5), and $\phi=82^{\circ}$ (II-9). For II-1, both the HOMO and LUMO are delocalized over both rings; for higher dihedral twist angles, as in II-5 the orbitals become more localized on one ring, with the HOMO localized on the phenyl ring while the LUMO is more localized on the pyridine ring. 
Insert Figure 4 here.

We have discussed the attractive, substitutionally tunable features of these simple twisted -conjugated merocyanine systems. The principal factor affecting the linear and NLO response in these chromophores is the ease with which charge separation can be effected and how the charge separation is stabilized. Even though the NLO responses of these seemingly simple chromophores are very attractive, the experimental realization is less than trivial. As a design motif, however, this controlled twist, and therefore controlled mixing of quinoid and charge-separated forms seems both powerful and useful, and is reminiscent of the related bond-length alternation scheme [20] as a conceptual design tool.

\section{Mobility calculations in conducting oligomers}

A key challenge for understanding conductivity of organic $\pi$-electronic materials is to characterize those structural factors important in charge transfer, including both charge mobility and majority carrier type. Particularly noteworthy is the frequently observed anisotropic character of the conductivity, [21,22] which implies preferred directions for maximum mobility in such materials and suggests that appropriate crystal engineering to enhance $\pi$-overlap between molecules may be an attractive route to improving the intrinsic mobility, for example by suppressing the typical herringbone packing of oligothiophenes in favor of parallel eclipsed orientations. Also noteworthy is the observed experimental crossover from p-type to n-type majority carrier behavior in substituted oligothiophene OFETs. [23,24] 
The charge-transfer process (for holes or electrons) between spatially-separated molecules (i.e., either two oligomers or two polymer chains) can be summarized by either of the following reactions:

$$
\begin{aligned}
& M+M^{+} \rightarrow M^{+}+M \\
& M+M^{-} \rightarrow M^{-}+M
\end{aligned}
$$

where $\mathrm{M}$ represents the molecule undergoing charge transfer, and the $\mathrm{M}^{+}$or $\mathrm{M}^{-}$species contain either the hole or electron, respectively. If the temperature is sufficiently high to treat vibrational modes classically, previous work yields the following Marcus-type expression for the hole (or electron) charge-transfer rate: [25-30]

$$
k_{\text {transfer }}=\left(\frac{\pi}{\lambda k_{b} T}\right)^{1 / 2} \frac{V^{2}}{\hbar} \exp \left(-\frac{\lambda}{4 k_{b} T}\right)
$$

where $\mathrm{T}$ is the temperature, $\hbar$ is the Planck constant, $k_{b}$ is the Boltzmann constant, and two parameters of key importance in hopping transport are the reorganization energy $(\lambda)$ accompanying geometric relaxation associated with the charge transfer, and the effective electronic coupling matrix element $(\mathrm{V})$ between neighboring species, dictated largely by orbital overlap. The latter is spatially-dependent, since it describes the overlap of particular orbitals dictated by the mutual separations and orientations of adjacent molecules.

We will approximate the electronic coupling matrix element with tight-binding theory and consider the orbital energy splitting for $\pi$-stacked dimers of oligothiophenes, oligofurans, and oligopyrroles (Figure 5). While experimental single-crystal structures are unknown for the oligofurans and oligopyrroles, it is reasonable to assume that they adopt structures similar to those observed in oligothiophene crystal structures (e.g., 
Figure 6 for tetrathiophene [31]). In particular, oligothiophenes crystallize in herringbone motifs (e.g., extending along the $b$ axis as shown in Figure 6) rather than in cofacial $\pi$ stacks. Across the herringbone stacks (e.g., extending along the " $d$ " diagonal axis in Figure 6 ), are tilted $\pi$-stacks. The herringbone and tilted motifs resemble the slipped stacking and T-shaped motifs observed in benzene $\pi$-dimers. [32]

Insert Figure 5,6 and Chart 3 here.

Table 3 and 4 give the DFT-computed internal reorganization energies and MP2computed orbital splitting energies for the oligothiophenes, oligopyrroles, and oligofurans considered, using geometries modeling the crystal packing observed for oligopyrroles and oligofurans. These can be used to calculate the total hole or electron charge transfer rates (Figure 7). Most notably, the large increases in charge transfer rates as a function of increasing oligomer length are driven largely by the decrease in internal reorganization energies - suggesting that this is a key parameter for understanding intrinsic ambient-temperature charge transfer rates in conducting/semiconducting organic solids, due to the inverse exponential $\mathrm{e}^{-/ 4 \mathrm{kT}}$ term in the charge transfer rate. [25]

Insert Figure 7 here. 


\section{Charge injection barriers}

The performance and operation of electronic devices based on organic semiconductors are strongly dependent on how charges are injected from the conducting element(s) (metals, transparent conducting oxides, conducting polymers) to the active organic material(s).[33] Besides the physical nature of the conductor/semiconductor interface, the first order parameter governing such a process is the energy difference between the conductor workfunction and the semiconductor frontier molecular orbitals (HOMO and LUMO). Electron/hole injection requires electron/hole transfer from the Fermi level of the conductor to the semiconductor LUMO/HOMO (This orbital picture is a convenient short-hand: what is actually involved are molecular cation/anion states). Indeed, a number of studies have demonstrated that energy level matching is the key parameter to improved device performance, stability, and reliability. To this end, the Marks group has been providing fundamental contributions, in particular in the area of organic light-emitting diodes (OLEDs) and field-effect transistors (OFETs), Figure 8. [34]

Insert Figure 8 here.

Here we present an overly simple but effective model for the charge injection across a metal/organic interface for the oligothiophene series $\mathbf{1}-\mathbf{5}$ (Figure 9). We employ computed orbital energies in a Schottky barrier model to describe the relative injection characteristics of electrons and holes. In our ultra-simple model, we are 
assuming that the reduction and oxidation energies are given by the LUMO and HOMO orbitals, respectively. This is equivalent to assuming the validity of Koopmans' theorem, which is not valid in any Kohn-Sham level (single determinant) electronic structure model (except for the HOMO level). [35]. In practice, however, these computed orbital energies do correspond rather well [36] to the (vertical) electron affinities and ionization energies, at least for the small organics considered here. We find that the results compare favorably with recent experimental data for electron and hole transport in oligothiophenebased devices. [37]

Insert Figure 9 here.

The simplest picture for a Schottky-type injection barrier for hopping-type carrier transport (e.g., over a metal/organic interface described above) can be described by a Boltzmann-like distribution, [38,39] (Figure 10) and expressed as:

$$
\text { rate } \propto e^{-\Delta E / k_{b} T}
$$

where $\Delta \mathrm{E}$ is the magnitude of the injection barrier, $\mathrm{T}=298 \mathrm{~K}$, and $k_{\mathrm{b}}$ is the Boltzmann constant. Although Equation 3 is a simplified picture of charge injection since it does not explicitly incorporate space charge, image effects or interfacial dipole layers, and holds approximately only for $\Delta \mathrm{E}>0$, experimental data show it to describe the properties of metal/organic interfaces, e.g., in OFETs or OLEDs, reasonably well, [38,39] and it is physically reasonable to assume that these effects remain essentially constant across a related series of compounds. Unsubstituted and alkyl-/perfluoroalkyl-substituted 
oligothiophenes $\mathbf{1}-\mathbf{5}$ are ideal candidates for this study (Figure 9) since the HOMO and LUMO spatial compositions are essentially constant and packing/stacking characteristics (from crystal structure) as well as thin-film microstructure are very similar across the series [40]).

With this exponential dependence of carrier injection rate on $\Delta \mathrm{E}$, differences of a few tenths of an $\mathrm{eV}$ in the barrier height can significantly alter the injection characteristics. Therefore, within the assumptions stated above, the relative carrier injection barriers should be describable as the difference between the molecular HOMO energy and the metal electrode work function for hole injection, and the difference between the molecular LUMO energy and the metal electrode work function for electron injection. Using this definition, we have computed hole and electron injection rates for a broad series of conductors relative to the HOMO or LUMO levels of unsubstituted $\alpha 6 \mathrm{~T}$, and representative results are summarized in Table 5. The molecule $\alpha 6 \mathrm{~T}$ was chosen as a model since it is (micro)structurally similar to the other oligothiophenes and it exhibits ptype activity in a variety of semiconductor-based devices such as FETs, OLEDs, and solar cells [41].

Insert Figure 10 and Table 5 here.

From the relative HOMO and LUMO energies for $\mathbf{1}$ - $\mathbf{5}$ clear trends in the charge injection rates are apparent relative to 6T. While only hole injection ( $p$-type conduction) is computed for unsubstituted $\mathrm{nT}$ molecules $\mathbf{5}$, the approximately $0.4 \mathrm{eV}$ difference in 
barrier heights for DFH-6T (found $n$-type in a FET structure) relative to 6T ( $p$-type) results in a predicted decrease of over six orders of magnitude in the hole injection rate and a similar increase in electron injection rate. While computed changes in injection rate greater than several orders of magnitude are only suggestive, Table 5 clearly indicates that the changeover from hole to electron injection/transport occurs with a modest $\sim 2$ or 3 orders of magnitude increase in electron injection rate over that of 6T. Moreover, the enhancement in electron injection is often accompanied by a decrease in hole injection (Table 5), so that a complete inversion in majority carrier type is predicted (and found experimentally for many of these materials in both OFET/OLED devices) to occur. Another useful metric is the ratio between the relative hole- and electron-injection rates given in Table 5. Here both computed rates are taken relative to the standard, eliminating the effect of the standard on the rate ratio. Since, often, both frontier orbitals move in the same way (up or down, energetically) upon substitution, increase in one rate is usually accompanied by the decrease of the opposing rate. The rate ratio provides a potential measure for the likely majority carrier due to injection rates. Using this ratio, it can be noted (for example) that the increase of relative electron injection rates of $\mathbf{1}$ and $\mathbf{2}(\mathrm{n}=2$ 6) are accompanied by a much larger decrease of hole injection rates, comparatively and vice versa, for the hole/electron injection rates of $\mathbf{3}-\mathbf{5}(n=2-6)$. Therefore the balance between these two parameters (the hole and electron injection rates) appears to drive the system toward exclusively n- or p-type activity. Furthermore, it suggests that from an energetic point of view, oligothiophenes with longer cores are more "labile" in terms of their carrier charge sign, hence an inversion between $p$ and $n$-type OFET activity is more likely in 6Ts than in the corresponding 2Ts derivatives. 
As noted above, the present Schottky barrier model does not explicitly treat electrostatic and covalent effects on the metal-organic charge injection barrier which could, in principle, be significant. Thus, an intrinsic electron injection barrier of $\sim 2.8 \mathrm{eV}$ is estimated for a DFH-nT-Au interface (assuming a $5.4 \mathrm{eV}$ Au work function [42]) in the absence of level shifting. However, such effects are reasonably assumed to be constant over a closely related series of molecules, and indeed this is supported by experimental UPS data, evidencing a uniform vacuum level shift across metal-oligothiophene interfaces. [43] In summary, the present results demonstrate that a simple Schottky barrier model provides excellent predictive guidance as to the majority carrier type of widely studied organic conductors such as oligothiophenes, arguing also that the majority carrier sign measured in OLED's or transport junctions of such materials is largely governed by injection characteristics. Future refinements will focus on including in this model the analysis/prediction of absolute metal-organic interfacial barrier heights and interfacial dipole layer [44] effects.

\section{Concluding remarks}

One of the major challenges in the area of organic electronics and optoelectronics is mechanism-based design of optimized materials. In this contribution, we have examined three important processes: nonlinear optical molecular response, mobility in oligomeric conductors and charge injection at the OFET electrode/organic interface. For each, we have used appropriate but very simple models to optimize and to understand the response.

The controlled-twist motif discussed in section II represents a new but promising design approach to nonlinear modulators and interferometers. The mobility calculations of 
section III suggest both optimal molecular structure and crystal packing for charge transport. The Schottky injection model of section IV used a very simple approach to predict, quite accurately, the relative injection efficiencies, and therefore the transport type, for molecular conductors.

\section{Acknowledgements:}

We are grateful to the NSF/MRSEC program for support through the Northwestern MRSEC (grant DMR-0076077), and to the NASA Institute for Nanoelectronics and Computing (Award NCC 2-3163) for support. This paper is in honor of Tobin Marks treasured friend, teacher, colleague, inspirer and leader. Tobin's remarkable insights into how molecular matter behaves have been honored by many awards, but the coherence, creativity, breadth and depth of his science speak plainly for themselves. 


\section{Appendix A}

The geometry of all chromophores was calculated with the DFT/B3LYP [45], [46] suite in Jaguar [47] with a $6-31 \mathrm{G}^{* *}$ basis set. For the molecules of Table 1 , the twist angle $\varphi$ around the double bond (Chart 1) was constrained, while all the other degrees of freedom were optimized without restraint.

The excitation energies and NLO responses were calculated using the semiempirical intermediate neglect of differential overlap (INDO) Hamiltonian [48] coupled to the multireference single and double configuration interaction technique (MRD-CI) [14] [24].

As reference determinants, we chose the ones dominant in the description of the ground state and the lowest-lying excited state, as a proper description of these states is critical for the calculation of second-order response properties in the studied molecules. For both chromophore sets, I and II, the reference determinants include the selfconsistent field (SCF, no excitation) RHF singlet determinant, the singly (HOMO to LUMO) excited determinant, and the determinant described by the double excitation from the HOMO to the LUMO. For the II chromophore series, we also included the singly excited determinant that corresponds to electron excitation from the HOMO to the LUMO+4 (contributes to the description of the electronic structure of the quinoid form, small twist angle) and the determinant corresponding to electron excitation from the HOMO to the LUMO+2 (contributes to the description of the charge-separated form, large twist angle). For reference determinants of single excitations the CI active space consisted of the HOMO and 15 orbitals below and 15 orbitals above the HOMO. For 
reference determinants of double excitations the CI space consisted of the HOMO and 5 orbitals below and above the HOMO at small twist angles.

The second-order polarizability $\beta$ appears as a third-rank tensor in the first nonlinear term that arises in the dependence of the molecular dipole moment $\mu$ on the electric field $F$ experienced by the molecule [49]:

$$
\mu_{i}=\mu_{i}^{(0)}+\sum_{i} \alpha_{i j} F_{j}+1 / 2 \sum_{j, k} \beta_{i j k} F_{j} F_{k}
$$

Here, $\mu_{i}^{(0)}$ is the permanent dipole moment of the molecule in direction $i ; F_{j}$ is the component of the electromagnetic field in the $j$ direction; $\alpha$ and $\beta$ are the static (zero frequency, $\omega=0.0 \mathrm{eV}$ ) first- and second- order polarizability tensors. We are interested in $\beta_{n}\left(\beta_{\text {vector }}\right)$ component of the $\beta$ tensor:

$$
\beta_{\mu}=\frac{\sum_{i=x, y, z} \beta_{i} \mu_{i}}{\|\mu\|} \quad\left(\beta_{i}=\frac{1}{3} \sum_{k=x, y, z}\left(\beta_{i k k}+\beta_{k i k}+\beta_{k i k}\right)\right)
$$

Calculations of the second order polarizability were performed using the sum-over-states (SOS) [50] formalism with the excited state energies, dipole moments and transition dipole calculated either with the MRD-CI or the CIS approaches. 


\section{Charts, tables and figures:}

Table 1. Computed excitation energy (in eV), and hyperpolarizability values $\left(\beta_{n}, 10^{-30}\right.$ esu) as a function of the dihedral twist angle about the central double bond for chromophore set $\mathbf{I}$, as calculated by the MRD-CI INDO/S methods.

Table 2. Optimized twist angle and computed excitation energy (in eV), hyperpolarizability values $\left(\beta_{n}, 10^{-30} \mathrm{esu}\right)$ as a function of the four substituents for chromophore set II, as calculated by DFT and the MRD-CI INDO/S methods.

Table 3. DFT-computed internal reorganization energies (in $\mathrm{eV}$ ) for oligothiophenes, oligofurans, and oligopyrroles $(n=2-4)$ for both hole transfer $\left(\lambda_{+}\right)$and electron transfer $(\lambda)$.

Table 4. MP2-computed orbital splitting (in eV) for oligothiophenes, oligofurans, and oligopyrroles $(n=2-4)$ calculated from oligomer pairs in a given spatial orientation for both hole transfer (HOMO) and electron transfer (LUMO). Orientations were chosen as representative of oligothiophene crystal structures.

Table 5. Summary of representative computed electronic structures, relative to $\mathrm{DFHCH}_{2}{ }^{-}$ $6 \mathrm{~T}$ with HOMO and LUMO energies in $\mathrm{eV}$, and relative hole $\left(\mathrm{R}^{\mathrm{h}+}\right)$ and electron $\left(\mathrm{R}^{\mathrm{e}-}\right)$ injection rates and charge injection rate ratio given in orders of magnitude difference.

Chart 1. Chromophore set I.

Chart 2. Chromophore set II.

Chart 3. Three $\pi$-stacking motifs.

Figure 1. Variation of the excitation energy to the lowest-lying excited state (Left side) and $\beta_{\mu}$ (Right side) as a function of the molecular twist angle $\varphi$ for chromophore set $\mathbf{I}$. 
Figure 2. Protection-deprotection self-assembly scheme for surface functionalization with large hyperpolarizability chromophores. In order to bind the chromophores to the surfaces, two different linkers are used.

Figure 3. Variation of the excitation energy to the lowest-lying excited state (Left side) and $\beta \mu$ (Right side) as a function of the molecular twist angle $\varphi$ for chromophore set II. Figure 4. INDO/S derived HOMO and LUMO molecular orbitals for II-1, II-5 and II-9. The shading represents the phase of the wavefunctions.

Figure 5. Heterocyclic oligomers studied, including oligothiophenes (a), oligofurans (b), and oligopyrroles (c) of varying lengths $(n=2-4)$. Also shown are the structures of $C_{60}$ and NTCDA as example organic $n$-type conductors.

Figure 6. Experimental single-crystal packing of tetrathiophene (ref. [49]), showing a $\pi$ herringbone stacking (slipped-stacked) motif extending along the $b$ axis, and along the diagonal " $d$ " axis between herringbone stacks as tilted $\pi$-stacks.

Figure 7. Total hole and electron transfer rates for oligothiophenes ( $\mathbf{\Lambda})$, oligofurans ( $\mathbf{(})$, and oligopyrroles $(\bullet)$ from eq. 2 , using the internal reorganization energies $(\lambda)$ calculated for hole and electron transfer from reference [25] and Table 5, and splitting computed with $\pi$-stacking orientations of tilted and slipped-stack herringbone motifs with geometric parameters approximate various oligothiophene crystal structures.[25]

Figure 8. Schematic representation of OLED and OFET devices.

Figure 6. Families of oligothiophenes studied.

Figure 10. A. Schematic of Schottky-type barrier between a metal and organic semiconductor, showing electron and hole injection barriers and the HOMO and LUMO 
level configuration for oligothiophenes. $\alpha, \omega$-substitution or $\beta, \beta$ '-substitution do not significantly change the spatial distributions of these orbitals. Note the different characteristics of the HOMO and LUMO. B. Plots of the computed molecular orbital energetics of oligothiophene families $\mathbf{1}$ - $\mathbf{5}$ studied vs. the number of heterocycle rings in the oligomer. DFH-nTs 1 and isoDFH-nTs $2(\bullet)$, DH-nTs 3 and isoDH-nTs $4(\mathbf{\Delta})$, anT 5 $(\star)$. 
Table 1. Computed excitation energy (in $\mathrm{eV})$, and hyperpolarizability values $\left(\beta_{\mu}, 10^{-30}\right.$ esu) as a function of the dihedral twist angle about the central double bond for chromophore set I, as calculated by the MRD-CI INDO/S methods.

\begin{tabular}{|l|r|r|r|}
\hline Chromophore & $\begin{array}{c}\text { Twist } \\
\text { dihedral } \\
\text { Angle } \\
\text { [degrees] }\end{array}$ & $\begin{array}{c}\text { Excitation } \\
\text { energy } \\
{[\mathrm{eV}]}\end{array}$ & $\begin{array}{l}\beta_{\mu} \\
{\left[* 10^{-30}\right.} \\
\text { esu }]\end{array}$ \\
\hline $\mathrm{I}-20$ & 20 & 2.60 & 17.1 \\
\hline $\mathrm{I}-30$ & 30 & 2.41 & 18.7 \\
\hline $\mathrm{I}-40$ & 40 & 2.14 & 21.8 \\
\hline $\mathrm{I}-45$ & 45 & 1.98 & 23.8 \\
\hline $\mathrm{I}-50$ & 50 & 1.80 & 26.0 \\
\hline $\mathrm{I}-57$ & 57 & 1.54 & 29.9 \\
\hline $\mathrm{I}-60$ & 60 & 1.40 & 29.4 \\
\hline $\mathrm{I}-65$ & 65 & 1.19 & 24.9 \\
\hline $\mathrm{I}-70$ & 70 & 0.97 & -3.5 \\
\hline $\mathrm{I}-75$ & 75 & 0.76 & -250.8 \\
\hline $\mathrm{I}-80$ & 80 & 0.60 & -772.7 \\
\hline $\mathrm{I}-85$ & 85 & 0.54 & -731.2 \\
\hline $\mathrm{I}-90$ & 90 & 0.55 & -25.2 \\
\hline
\end{tabular}


Table 2. Optimized twist angle and computed excitation energy (in eV), hyperpolarizability values $\left(\beta_{n}, 10^{-30} \mathrm{esu}\right)$ as a function of the four substituents for chromophore set II, as calculated by DFT and the MRD-CI INDO/S methods.

\begin{tabular}{|c|c|c|c|c|c|c|c|}
\hline Chromophore & $\mathrm{X} 1$ & $\mathrm{X} 2$ & Y1 & Y2 & $\begin{array}{c}\text { Equilibrium } \\
\text { twist } \\
\text { dihedral } \\
\text { Angle } \\
\text { [degrees] }\end{array}$ & $\begin{array}{c}\text { Excitation } \\
\text { energy } \\
{[\mathrm{eV}]}\end{array}$ & {$\left[\begin{array}{l}\beta_{\mu} \\
{\left[* 10^{-30}\right.} \\
\text { esu }]\end{array}\right.$} \\
\hline II-1 & $\mathrm{H}$ & $\mathrm{H}$ & $\mathrm{CH} 3$ & $\mathrm{CH} 3$ & 35 & 2.23 & 78.0 \\
\hline II-2 & $\mathrm{H}$ & $\mathrm{H}$ & Et & Et & 38 & 2.16 & 71.0 \\
\hline II-3 & $\mathrm{H}$ & $\mathrm{CH} 3$ & $\mathrm{CH} 3$ & $\mathrm{CH} 3$ & 48 & 1.76 & 43.8 \\
\hline II-4 & $\mathrm{CH} 3$ & $\mathrm{CH} 3$ & $\mathrm{H}$ & $\mathrm{CH} 3$ & 50 & 1.76 & -42.4 \\
\hline II-5 & $\mathrm{CH} 3$ & $\mathrm{CH} 3$ & $\mathrm{CH} 3$ & $\mathrm{CH} 3$ & 60 & 1.37 & -287.4 \\
\hline II-6 & $\mathrm{CH} 3$ & $\mathrm{H}$ & $\mathrm{t}-\mathrm{Bu}$ & $\mathrm{t}-\mathrm{Bu}$ & 70 & 1.00 & -985.0 \\
\hline II-7 & $t-B u$ & $t-B u$ & $\mathrm{H}$ & $\mathrm{CH} 3$ & 72 & 1.01 & -1328.9 \\
\hline II-8 & $\mathrm{CH} 3$ & $\mathrm{CH} 3$ & $\mathrm{t}-\mathrm{Bu}$ & $\mathrm{t}-\mathrm{Bu}$ & 76 & 0.92 & -1475.3 \\
\hline II-9 & $\mathrm{t}-\mathrm{Bu}$ & $\mathrm{t}-\mathrm{Bu}$ & $\mathrm{t}-\mathrm{Bu}$ & $t-B u$ & 82 & 1.02 & -500.1 \\
\hline
\end{tabular}


Table 3. DFT-computed internal reorganization energies (in $\mathrm{eV}$ ) for oligothiophenes, oligofurans, and oligopyrroles $(n=2-4)$ for both hole transfer $\left(\lambda_{+}\right)$and electron transfer $\left(\lambda_{-}\right)$

\begin{tabular}{ccccccc}
\hline $\begin{array}{c}\text { \# of } \\
\text { monomer }\end{array}$ & \multicolumn{2}{c}{ Oligothiophenes } & \multicolumn{2}{c}{ Oligofurans } & \multicolumn{2}{c}{ Oligopyrroles } \\
units & $\lambda_{+}$ & $\lambda_{-}$ & $\lambda_{+}$ & $\lambda_{-}$ & $\lambda_{+}$ & $\boldsymbol{\lambda}_{-}$ \\
\hline $\mathbf{2}$ & 0.365 & 0.331 & 0.315 & 0.394 & 0.353 & 0.402 \\
$\mathbf{3}$ & 0.321 & 0.286 & 0.292 & 0.324 & 0.312 & 0.319 \\
$\mathbf{4}$ & 0.292 & 0.259 & 0.271 & 0.286 & 0.281 & 0.281 \\
\hline
\end{tabular}

Table 4. MP2-computed orbital splittings (in eV) for oligothiophenes, oligofurans, and oligopyrroles $(n=2-4)$ calculated from oligomer pairs in a given spatial orientation for both hole transfer (HOMO) and electron transfer (LUMO). Orientations were chosen as representative of oligothiophene crystal structures.

\begin{tabular}{|c|c|c|c|c|c|c|c|}
\hline \multirow{2}{*}{$\begin{array}{c}\text { Spatial } \\
\text { Orientation }\end{array}$} & \multirow{2}{*}{$\begin{array}{c}\# \text { of } \\
\text { monomer } \\
\text { units }\end{array}$} & \multicolumn{2}{|c|}{ Oligothiophenes } & \multicolumn{2}{|c|}{ Oligofurans } & \multicolumn{2}{|c|}{ Oligopyrroles } \\
\hline & & НОМО & LUMO & НОМО & LUMO & HOМO & LUMO \\
\hline Tilted & 2 & 0.244 & 0.225 & 0.233 & 0.291 & 0.321 & 0.353 \\
\hline $\begin{array}{l}60^{\circ} \text { tilt } \\
5.0 \AA\end{array}$ & 3 & 0.232 & 0.229 & 0.224 & 0.296 & 0.447 & 0.452 \\
\hline $\begin{array}{c}\text { Z- } \\
\text { separation }\end{array}$ & 4 & 0.277 & 0.294 & 0.280 & 0.326 & 0.384 & 0.403 \\
\hline Slipped Stack & 2 & 0.010 & 0.292 & 0.045 & 0.071 & 0.037 & 0.117 \\
\hline $3.1 \AA / 4.84 \AA$ & 3 & 0.059 & 0.286 & 0.095 & 0.094 & 0.015 & 0.165 \\
\hline $\begin{array}{c}\text { Z/y } \\
\text { separation }\end{array}$ & 4 & 0.010 & 0.292 & 0.081 & 0.058 & 0.011 & 0.277 \\
\hline
\end{tabular}


Table 5. Summary of representative computed electronic structures, relative to $\mathrm{DFHCH}_{2}$ $6 \mathrm{~T}$ with HOMO and LUMO energies in $\mathrm{eV}$, and relative hole $\left(\mathrm{R}^{\mathrm{h}+}\right)$ and electron $\left(\mathrm{R}^{\mathrm{e}-}\right)$ injection rates and charge injection rate ratio given in orders of magnitude difference.

\begin{tabular}{cccccc}
\hline Compound & $\begin{array}{c}\text { Relative } \\
\text { HOMO } \\
\text { Energy }(\mathbf{e V})\end{array}$ & $\begin{array}{c}\text { Relative } \\
\text { LUMO } \\
\text { Energy }(\mathbf{e V})\end{array}$ & $\mathbf{l o g}_{\mathbf{1 0}}\left(\mathbf{R}^{\mathbf{h}+}\right)$ & $\mathbf{l o g}_{\mathbf{1 0}}\left(\mathbf{R}^{\mathbf{e}}\right)$ & $\log _{\mathbf{1 0}}\left(\frac{R^{h+}}{R^{e-}}\right)$ \\
\hline DFH-2T & -1.633 & -0.081 & -27.42 & 1.37 & -28.79 \\
DFH-3T & -1.088 & -0.217 & -18.28 & 3.65 & -21.94 \\
DFH-4T & -0.789 & -0.217 & -13.25 & 3.65 & -16.91 \\
DFH-5T & -0.571 & -0.326 & -9.60 & 5.48 & -15.08 \\
DFH-6T & -0.408 & -0.381 & -6.86 & 6.40 & -13.25 \\
isoDFH-2T & -1.796 & 0.381 & -30.17 & -6.40 & -23.76 \\
isoDFH-3T & -1.088 & 0.027 & -18.28 & -0.46 & -17.82 \\
isoDFH-4T & -0.789 & 0.027 & -13.25 & -0.46 & -12.79 \\
isoDFH-5T & -0.544 & -0.109 & -9.14 & 1.82 & -10.97 \\
isoDFH-6T & -0.381 & -0.109 & -6.40 & 1.82 & -8.22 \\
DH-2T & -0.354 & 1.170 & -5.94 & -19.66 & 13.72 \\
DH-3T & -0.082 & 0.653 & -1.37 & -10.97 & 9.60 \\
DH-4T & 0.027 & 0.381 & 0.46 & -6.40 & 6.86 \\
DH-5T & 0.082 & 0.218 & 1.37 & -3.66 & 5.03 \\
DH-6T & 0.109 & 0.109 & 1.83 & -1.83 & 3.66 \\
isoDH-2T & -0.381 & 1.225 & -6.40 & -20.57 & 14.17 \\
isoDH-3T & -0.109 & 0.708 & -1.83 & -11.89 & 10.06 \\
isoDH-4T & -0.109 & 0.490 & -1.83 & -8.23 & 6.40 \\
isoDH-5T & -0.027 & 0.300 & -0.46 & -5.03 & 4.57 \\
isoDH-6T & 0.136 & 0.109 & 2.29 & -1.83 & 4.12 \\
2T & -0.680 & 0.925 & -11.43 & -15.54 & 4.12 \\
3T & -0.354 & 0.490 & -5.94 & -8.23 & 2.29 \\
4T & -0.163 & 0.245 & -2.74 & -4.12 & 1.38 \\
5T & -0.082 & 0.082 & -1.37 & -1.37 & 0.00 \\
6T & 0.000 & 0.000 & 0.00 & 0.00 & 0.00 \\
& & & & &
\end{tabular}




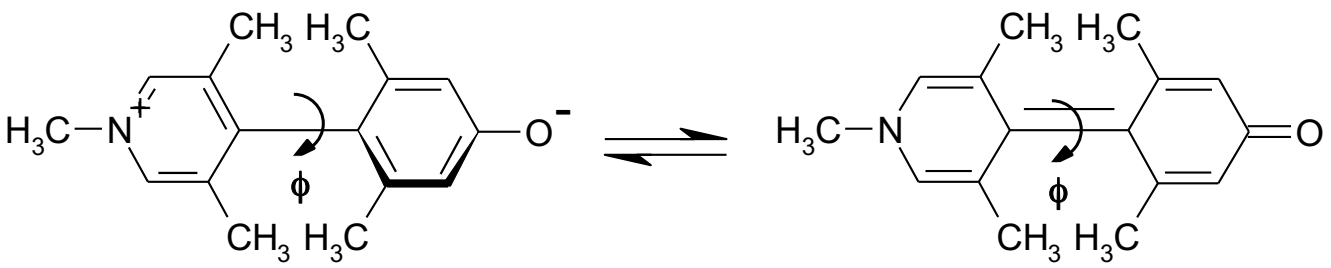

IA

IB

Chart 1

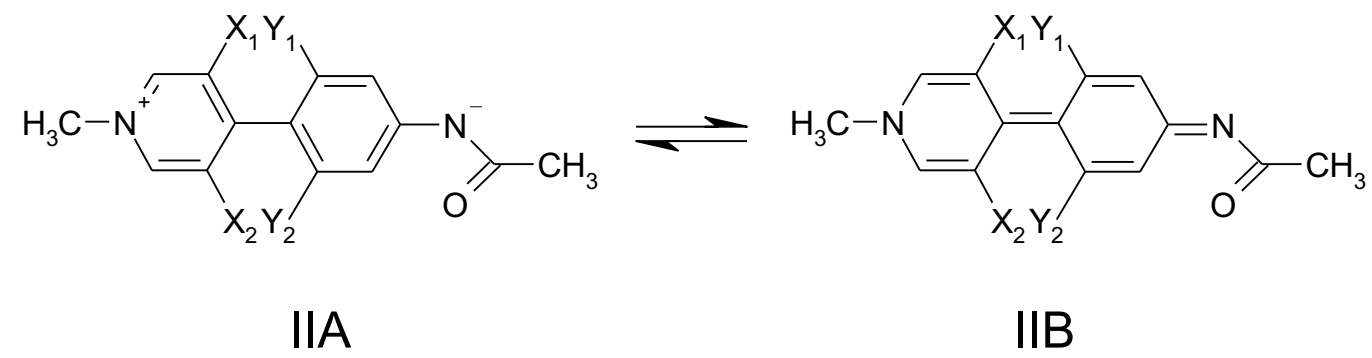

Chart 2 


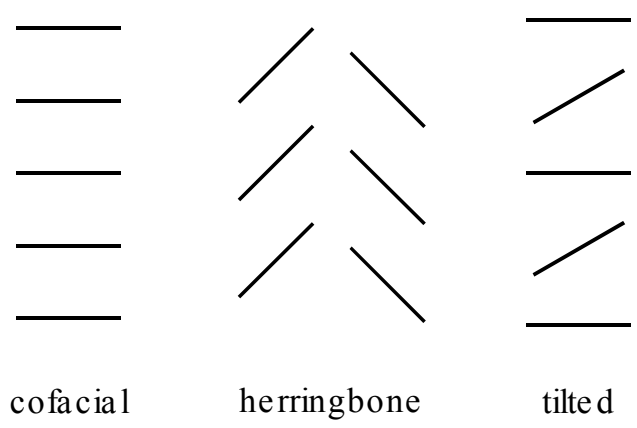

Chart 3 
Figure 1. Variation of the excitation energy to the lowest-lying excited state (left side) and $\beta \mu$ (right side) as a function of the molecular twist angle $\phi$ for chromophore set I.

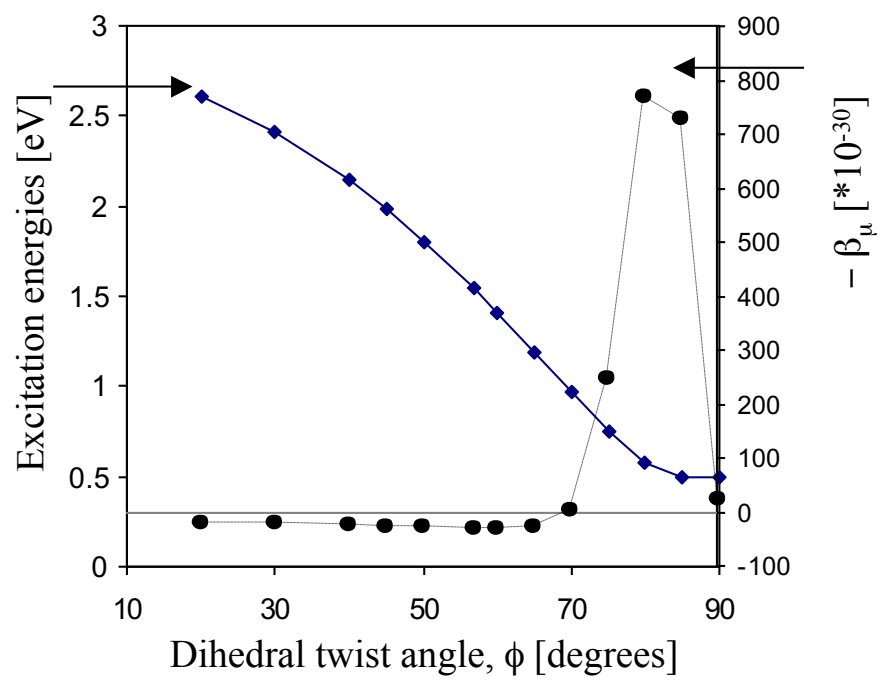


Fig. 2. Protection-deprotection self-assembly scheme for surface functionalization with large hyperpolarizability chromophores. In order to bind the chromophores to the surfaces two different linkers are used.
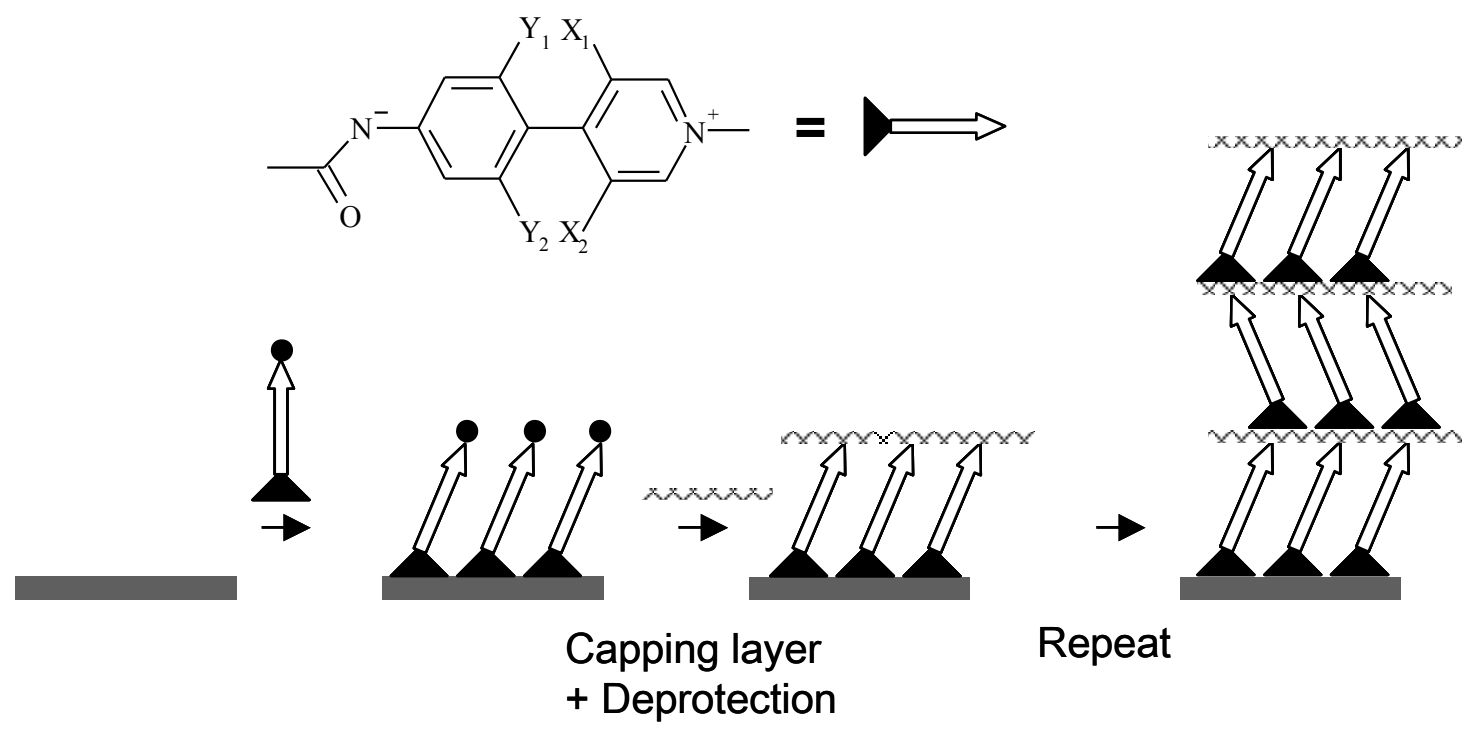
Figure 3. Variation of the excitation energy to the lowest-lying excited state (left side) and $\beta \mu$ (right side) as a function of the molecular twist angle $\phi$ for chromophore set II.

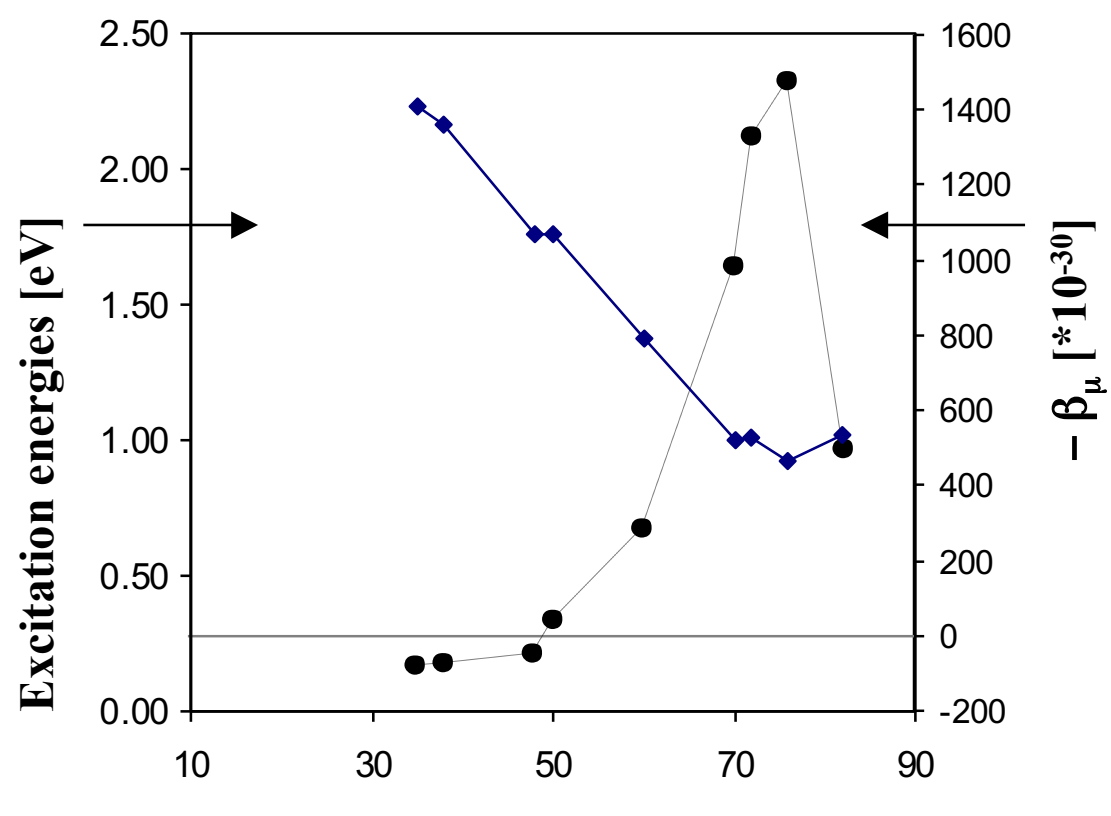

Dihedral twist angle, $\phi$ [degrees] 
Fig.4. INDO/S derived HOMO and LUMO molecular orbitals for II-1, II-5 and II-9. The

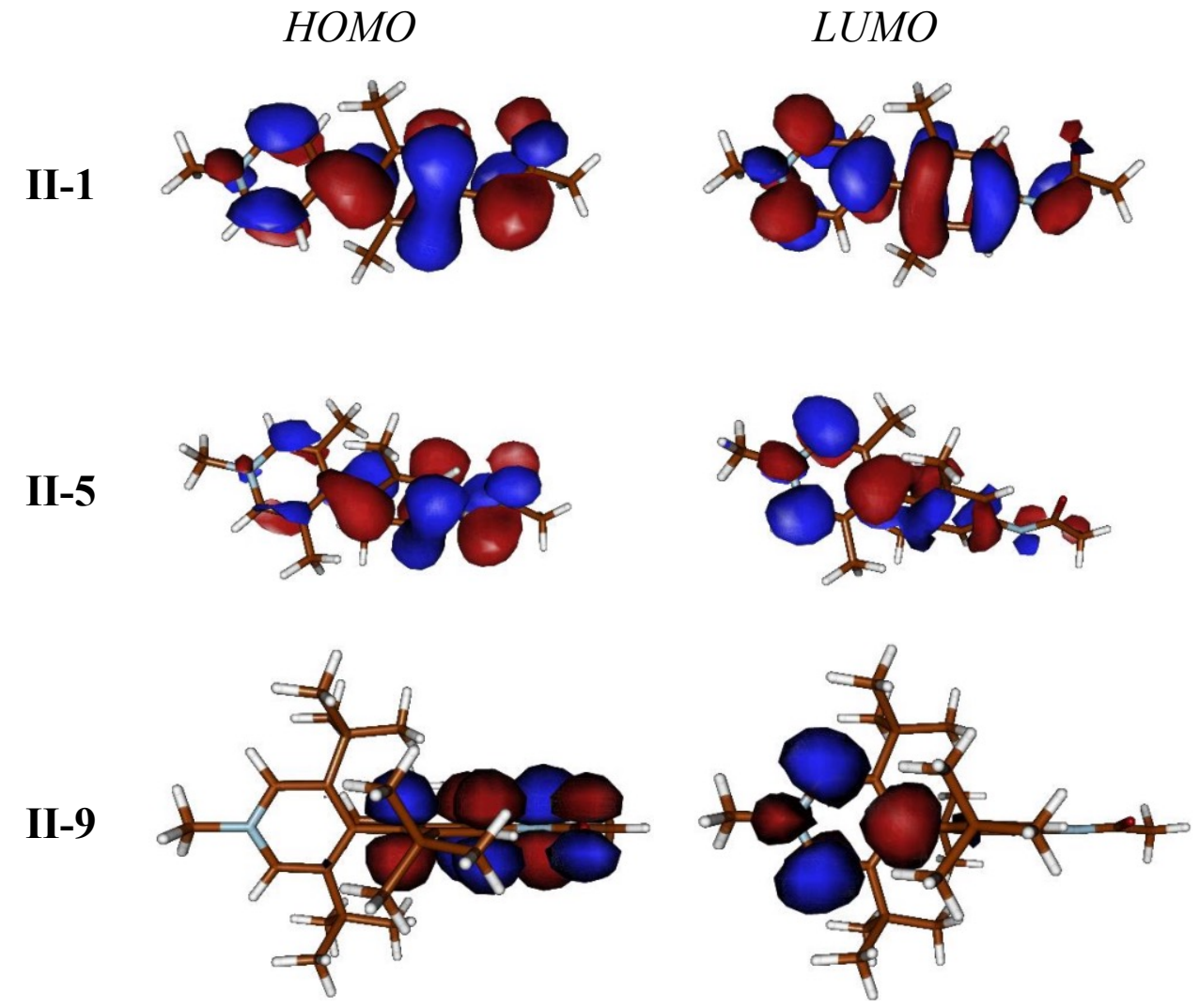

shading represents the phase of the wavefunctions. 
Figure 5. Heterocyclic oligomers studied, including oligothiophenes (a), oligofurans (b), and oligopyrroles $(\mathbf{c})$ of varying lengths $(n=2-4)$. Also shown are the structures of $\mathrm{C}_{60}$ and NTCDA as example organic $n$-type conductors.

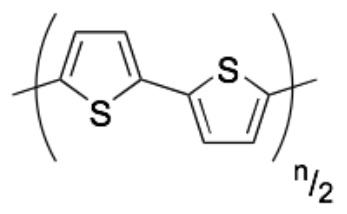

(a)

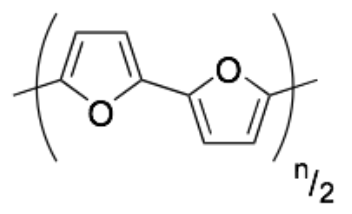

(b)

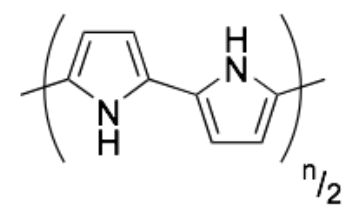

(c)

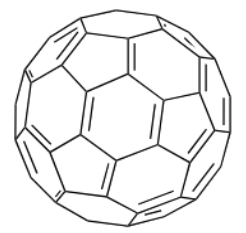

$\mathrm{C}_{60}$

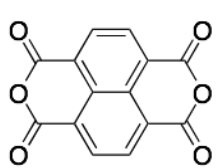

NTCDA 
Figure 6. Experimental single-crystal packing of tetrathiophene (ref. [51]), showing a $\pi$ herringbone stacking (slipped-stacked) motif extending along the $b$ axis, and along the diagonal " $d$ " axis between herringbone stacks as tilted $\pi$-stacks.
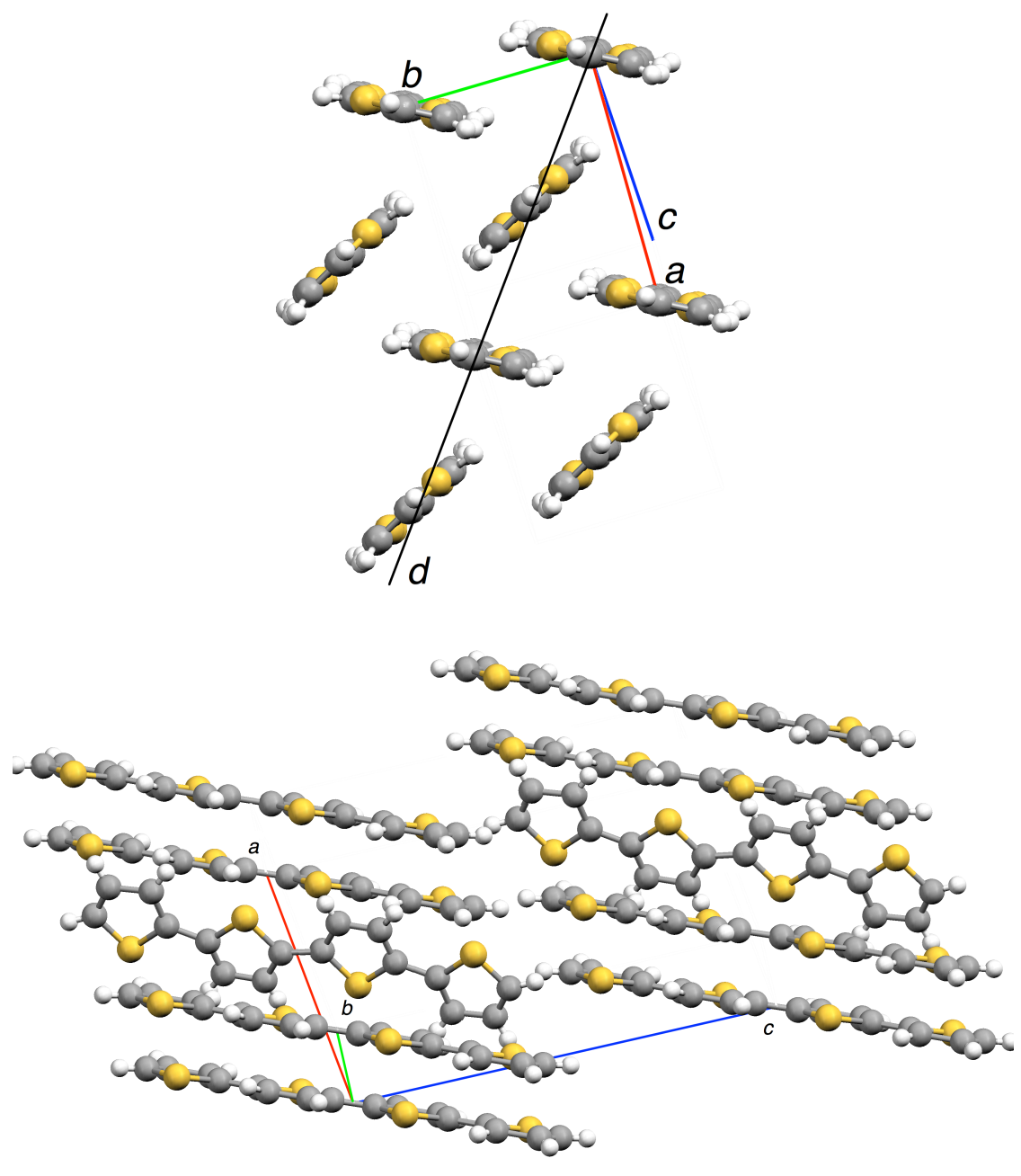
Figure 7. Total hole and electron transfer rates for oligothiophenes $(\triangle)$, oligofurans $(\square)$, and oligopyrroles $(\diamond)$ from eq. 2 , using the internal reorganization energies $(\lambda)$ calculated for hole and electron transfer from reference [25] and Table 5, and splitting computed with $\pi$-stacking orientations of tilted and slipped-stack herringbone motifs with geometric parameters approximate various oligothiophene crystal structures.[25]

Hole Transfer

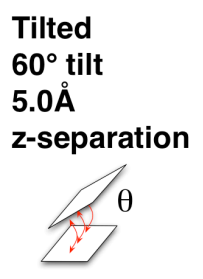

Slipped Stack $3.1 \AA ̊$ / $4.84 \AA$

$z / y$ separation

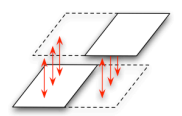

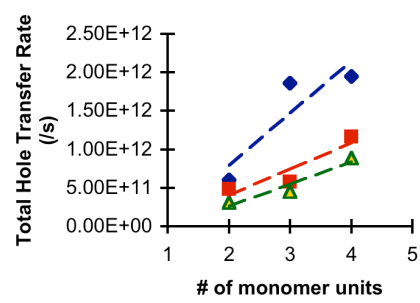

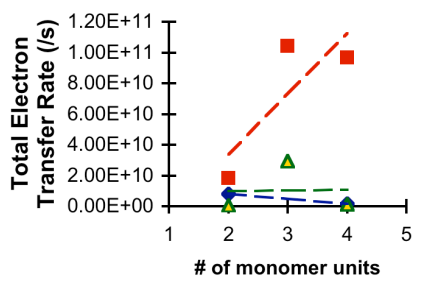

\section{Electron Transfer}
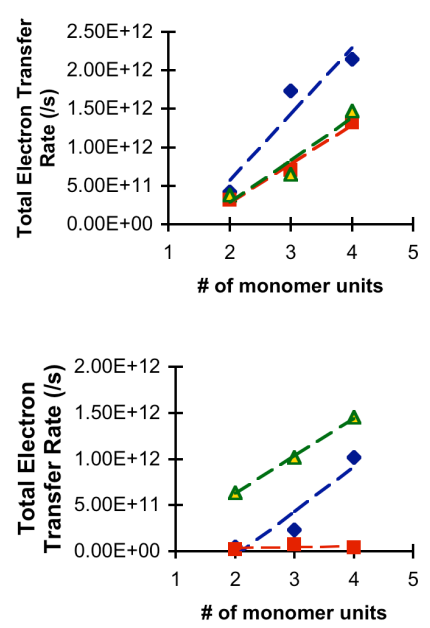
Figure 8. Schematic representation of OLED and OFET devices.

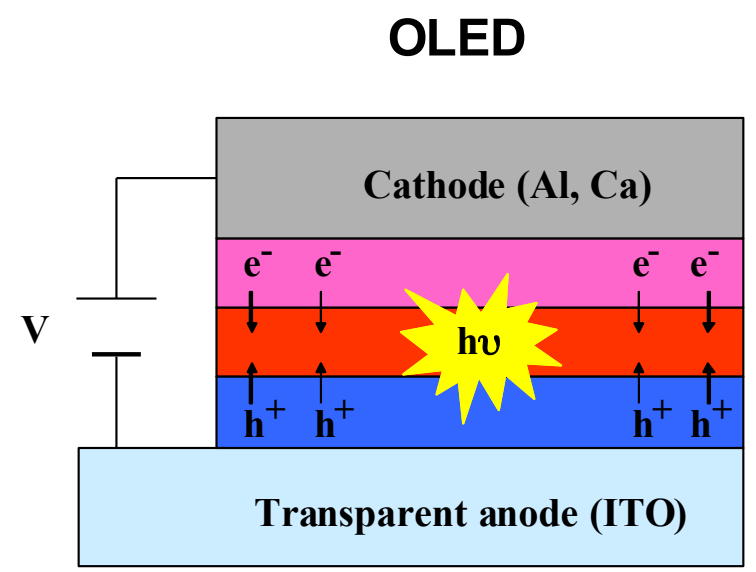

OFET

\begin{tabular}{|c|c|}
\hline Source & Drain \\
\hline Organic Semiconductor \\
\hline Dielectric \\
\hline Gate \\
\hline
\end{tabular}


Figure 9. Families of oligothiophenes studied.

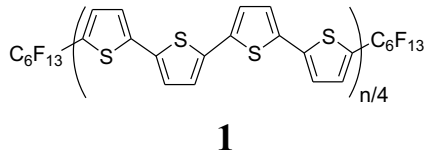

DFH-2T $: \mathrm{n}=2$

DFH-3T : $\mathrm{n}=3$

DFH-4T : $n=4$

DFH-5T : $n=5$

DFH-6T : $n=6$

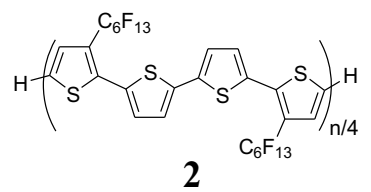

isoDFH-2T : $\mathrm{n}=2$

isoDFH-3T : $n=3$

isoDFH-4T : $n=4$

isoDFH-5T : $n=5$

isoDFH-6T $: \mathrm{n}=6$

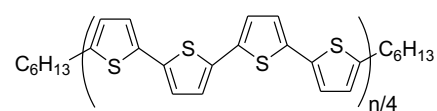

3

DH-2T : $\mathrm{n}=2$

DH-3T $: \mathrm{n}=3$

DH-4T: $\mathrm{n}=4$

DH-5T $: \mathrm{n}=5$

DH-6T $: \mathrm{n}=6$

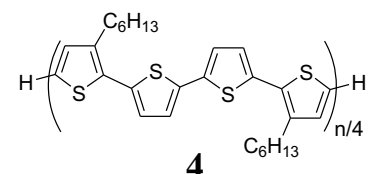

isoDH-2T : $\mathrm{n}=2$

isoDH-3T: $\mathrm{n}=3$

isoDH-4T : $\mathrm{n}=4$

isoDH-5T $: \mathrm{n}=5$

isoDH-6T $: \mathrm{n}=6$

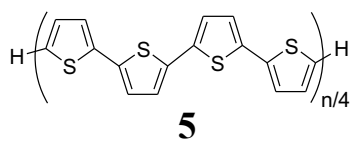

$\alpha 2 \mathrm{~T}: \mathrm{n}=2$

$\alpha 3 \mathrm{~T}: \mathrm{n}=3$

$\alpha 4 \mathrm{~T}: \mathrm{n}=4$

$\alpha 5 \mathrm{~T}: \mathrm{n}=5$

$\alpha 6 \mathrm{~T}: \mathrm{n}=6$ 
Figure 10. A. Schematic of Schottky-type barrier between a metal and organic semiconductor, showing electron and hole injection barriers and the HOMO and LUMO level configuration for oligothiophenes. $\alpha, \omega$-substitution or $\beta, \beta$ '-substitution do not significantly change the spatial distributions of these orbitals. Note the different characteristics of the HOMO and LUMO. B. Plots of the computed molecular orbital energetics of oligothiophene families $\mathbf{1}$ - $\mathbf{5}$ studied vs. the number of heterocycle rings in the oligomer. DFH-nTs 1 and isoDFH-nTs $2(\bullet)$, DH-nTs 3 and isoDH-nTs $4(\mathbf{\Delta})$, anT

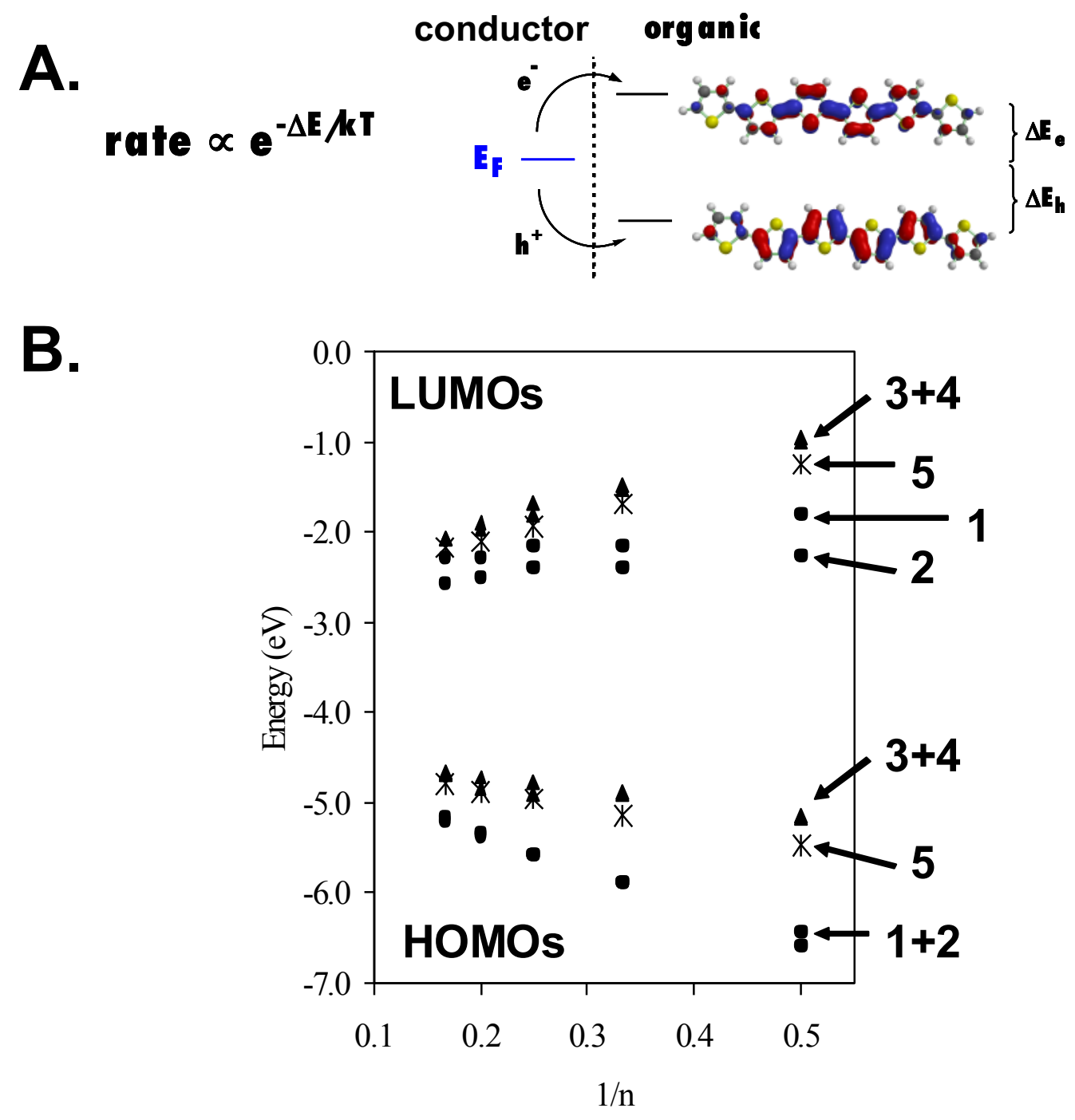


$5(\star)$.

- 39 - 


\section{References:}

[1] a) Metal-catalyzed Cross-coupling Reactions. F. Diederich, P. J. Stang, Eds. WileyVCH, Weinheim, (1998). b) D. J. Cardenas, Angew. Chem. Int. Ed. 42 (2003) 384. c) Handbook of Organopalladium Chemistry for Organic. E. -C. Negishi, (Ed) John Wiley \& Sons (2002).

[2] T. J. Marks, M. A. Ratner, Angew. Chem. Int. Ed. Eng. 34 (1995) 155.

[3] L. R. Dalton, J. Phys.-Cond. Mat. 15 (2003) R897.

[4] Poled polymers: a) P. M. Lundquist, S. Yitzchaik, T. J. Marks, G. K. Wong, S. DiBella, R. Cohen, G. Berkovic, Phys. Rev. B 55 (1997) 14055. b) P. M. Lundquist, M. Jurich, J. F. Wang, H. Zhou, T. J. Marks, G. K. Wong, App. Phys. Lett. 69 (1996) 901. Theoretical predictions: c) S. DiBella, I. Fragala, T.J. Marks, M.A. Ratner, J. Am. Chem. Soc. 118 (1996) 12747. d) I. D. L. Albert, T.J. Marks, M.A. Ratner, in "Progress in Organic Nonlinear Optics", M. Kuzyk, C. R. Dirk, Eds, (1998) MarcellDekker. e) S. Keinan, M. A. Ratner, T. J. Marks, Chem. Mater. (2004) ASAP Article. SAS: f) P. W. Zhu, H. Kang, A. Facchetti, G. Evmenenko, P. Dutta, T. J. Marks, J. Am. Chem. Soc. (2003) 125 11496. h) Y. G. Zhao, S. Chang, A. Wu, H. L. Lu, S. T. Ho, M. E. van der Boom, T.J. Marks, Opt. Eng. 42 (2003) 298. Device manufacturing: i) T. J. Marks, S. T. Ho, Z. Liu, P. Zhu, D. G. Sun, J. Ma, Y. Xiao, H. Kang, SPIE Proc. 4991 (2003) 133. j) Y. G. Zhao, A. Wu, H. L. Lu, S. Chang, W. K. Lu, S. T. Ho, M. E. van der Boom, T. J. Marks, App. Phys. Lett. 79 (2001) 587.

[5] For recent reviews of organic electro-optics, see: (a) A. Facchetti, A. Abbotto, L. Beverina, M. E. van der Boom, P. Dutta, G. Evmenenko, G. A. Pagani, T. J. Marks, Chem. Mater. 15 (2003) 1064. (b) P. Zhu, H. Kang, A. Facchetti, G. Evmenenko, P. Dutta, T. J. Marks, J. Am. Chem. Soc. 125 (2003) 11496. (c) M. E. van der Boom, Angew. Chem., Int. Ed. 18 (2002) 3363. (d) L. R. Dalton, J. Phys.-Cond. Matt. 15 (2003) R897. (e) J. Zyss, I. Ledoux-Rak, H. C. Weiss, D. Blaser, R. Boese, P. K. Thallapally, V. R. Thalladi, G. R. Desiraju, Chem. Mater. 15 (2003) 3063. (f) J. Zyss, Ed. "Molecular Nonlinear Optics: Materials, Phenomena and Devices" Chem. Phys. 245 (1999) (Special issue). (g) S. R. Marder, B. Kippelen, A. K. Y. Jen, N. Peyghambarian, Nature 388 (1997) 845. 
[6] M. E. Van Der Boom, P. Zhu, G. Evmenenko, J. E. Malinsky, W. Lin, P. Dutta, T. J. Marks, Langmuir 18 (2002) 3704.

[7] M. E. Van Der Boom, G. Evmenenko, P. Dutta, T. J. Marks, Adv. Funct. Mater. 11 (2001) 393.

[8] P. Ball, Made to measure, Princeton University Press, Princeton, 1997.

[9] I. D. L. Albert, T. J. Marks, M. A. Ratner, J. Am. Chem. Soc. 120 (1998) 11174.

[10] I. D. L. Albert, T. J. Marks, M. A. Ratner, J. Phys. Chem. 100 (1996) 9714.

[11] A. J. Kay, A. D. Woolhouse, G. J. Gainsford, T. G. Haskell, W. C. P., S. M. Giffin, I. T. McKinnie, T. H. Barnes, J. Mater. Chem. 11 (2001) 2271.

[12] S. Sitha, J. L. Rao, K. Bhanuprakash, B. M. Choudary, J. Phys. Chem. A 105 (2001) 8727.

[13] S. K. Pati, T. J. Marks, M. A. Ratner, J. Am. Chem. Soc. 123 (2001) 7287.

[14] D. Beljonne, J. Cornil, Z. Shuai, J.-L. Brédas, F. Rohlfing, D. D. C. Bradley, W. E. Torruellas, V. Ricci, G. I. Stegeman, Phys. Rev. B 55 (1997) 1505.

[15] S. Keinan, E. Zojer, J. L. Brédas, M. A. Ratner, T. J. Marks, THEOCHEM 633 (2003) 227.

[16] S. Keinan, E. Zojer, J. L. Brédas, M. A. Ratner, T. J. Marks, Unpublished results.

[17] M. A. Ratner, G. C. Schatz, Quantum Mechanics in Chemistry, Prentice-Hall, Upper Saddle River, NJ, 2000.

[18] D. R. Kanis, M. A. Ratner, T. J. Marks, Chem. Rev. 94 (1994) 195.

[19] a) P. Zhu, M. E. van der Boom, H. Kang, G. Evmenenko, P. Dutta, T. J. Marks, Chem. Mater. 14 (2002) 4982. b) Y. G. Zhao, A. Wu, H. L. Lu, S. Chang, W. K. Lu, S. T. Ho, M. E. van der Boom, T. J. Marks, App. Phys. Lett. 79 (2001) 587. c) W. Lin, W. Lin, G. K. Wong, T. J. Marks, J. Am. Chem. Soc. 118 (1996) 8034.

[20] S. R. Marder, L.-T. Cheng, B. G. Tiemann, A. C. Friedli, M. Blanchard-Desce, J. W. Perry, J. Skindhøj, Science 263 (1994) 511.

[21] R. Silerova, L. Kalvoda, D. Neher, A. Ferencz, J. Wu, G. Wegner, Chem. Mater. 10 (1998) 2284.

[22] P. N. Adams, P. J. Laughlin, A. P. Monkman, N. Bernhoeft, Sol. State Comm. 91 (1994) 875 .

[23] A. Facchetti, M. Mushrush, H. E. Katz, T. J. Marks, Adv. Mater. 15 (2003) 33. 
[24] A. Facchetti, Y. Deng, A. Wang, Y. Koide, H. Sirringhaus, T. J. Marks, R. H. Friend, (2000).

[25] Y. A. Berlin, G. R. Hutchison, P. Rempala, M. A. Ratner, J. Michl, J. Phys. Chem. A 107 (2003) 3970.

[26] P. F. Barbara, T. J. Meyer, M. A. Ratner, J. Phys. Chem. 100 (1996) 13148.

[27] R. A. Marcus, J. Phys. Chem. 24 (1956) 966.

[28] N. S. Hush, J. Phys. Chem. 28 (1958) 962.

[29] H. M. McConnell, J. Chem. Phys. 35 (1961) 508.

[30] R. A. Marcus, H. Eyring, Ann. Rev. Phys. Chem. 15 (1964) 155.

[31] L. Antolini, G. Horowitz, F. Kouki, F. Garnier, Adv. Mater. 10 (1998) 382.

[32] S. Tsuzuki, K. Honda, R. Azumi, J. Am. Chem. Soc. 124 (2002) 12200.

[33] a) D. Cahen, A. Kahn, Adv. Mater. 15 (2003) 271. b) A. Kahn, N. Koch, W.J. Gao, Polym. Sci., B: Polym. Phys. 41 (2003) 2529. c) S. J. J. Campbell, Vac. Sci. Tech. A. $21(2003) 251$.

[34] OLEDs: a) J. G. G. Veinot, H. Yan, S. M. Smith, J. Cui, Q. Huang, T. J. Marks, Nano Lett. 2 (2002) 333. b) Y. Koide, M. W. Such, R. Basu, G. Evmenenko, J. Cui, P. Dutta, M. C. Hersam, T. J. Marks, Langmuir 19 (2003) 86. c) H. Yan, Q. Huang, J. Cui, J. G. C. Veinot, M. Kern, T. J. Marks, Advan. Mater.15 (2003) 835. d) Y. Yang, Q. Huang, A. W. Metz, J. Ni, S. Jin, T. J. Marks, M. E. Madsen, A. DiVenere, S.- T. Ho, Adv. Mater. 16 (2004) 321. e) H. Yan, Q. Huang, B. J. Scott, T. J. Marks, Appl. Phys. Lett. 84 (2004) 3873. OFET: f) A. Facchetti, Y. Deng, A. Wang, Y. Koide, H. Sirringhaus, T. J. Marks, R. H. Friend, Angew. Chem., Int. Ed. 39 (2000) 4547. g) A. Facchetti, T. J. Marks, Polym. Prepr. 43 (2002) 734. h) A. Facchetti, M. Mushrush, H. E. Katz, T. J. Marks, Adv. Mater. 15 (2003) 15 33. i) A. Facchetti, M. H. Yoon, C. L. Stern, H. E. Katz, T. J. Marks, Angew. Chem., Int. Ed. 42 (2003) 3900. j) M. Mushrush, A. Facchetti, M. Lefenfeld, H. E. Katz, T. J. Marks, J. Am. Chem. Soc. 125 (2003) 9414. k) A. Facchetti, H. E. Katz, T. J. Marks, J. Veinot, in Organic Semiconductor Material, Gamota, D. Ed.; Kluwer Academic Publischer; Norwell, MA (2004).

[35] R. G. Parr, W. Yang, Density-Functional Theory of Atoms and Molecules, Oxford University Press, Oxford [England], 1994. 
[36] M. Levy, A. Nagy, Phys. Rev. A 59 (1999) 1687.

[37] Unpublished data from the Marks group indicate that OLEDs based on DFH-nT molecules as the electron-transporting material and DH-nTs molecules as holetransporting material exhibit efficient light emission. Note that devices based of the reverse configuration (DFH-nTs as hole-transporters and DH-nTs as electron transporters) do not function. This is a clear evidence that 1-5 exhibit complementary transport properties not only in FETs.

[38] A. K. Mahapatro, S. Ghosh, App. Phys. Lett. 80 (2002) 4840.

[39] A. B. Chwang, C. D. Frisbie, J. Phys. Chem. B 104 (2000) 12202.

[40] M. Jonsson, A. Houmam, G. Jocys, D. D. M. Wayner, J. Chem. Soc. -Perkin Trans. 2 (1999) 425.

[41] D. Fichou, J. Mater. Chem. 10 (2000) 571.

[42] Handbook of Chemistry and Physics, CRC Press, Boca Raton, Florida, 1995.

[43] a) H. Fujimoto, U. Nagashima, H. Inokuchi, K. Seki, Y. Cao, H. Nakahara, J. Nakayama, M. Hoshino, K. Fukuda, J. Chem. Phys. 92 (1990) 4077 b) A. J. Makinen, I. G. Hill, M. Kinoshita, T. Noda, Y. Shirota, Z. H. Kafafi, J. Appl. Phys. 91 (2002) 5456 c) A. J. Makinen, I. G. Hill, T. Noda, Y. Shirota, Z. H. Kafafi, Appl. Phys. Lett. 78 (2001) 670.

[44] X. Crispin, V. Geskin, A. Crispin, J. Cornil, R. Lazzaroni, W. Salaneck, J.-L. Brédas, J. Am. Chem. Soc. 124 (2002) 8131.

[45] A. D. Becke, Phys. Rev. A 38 (1988) 3098.

[46] C. Lee, W. Yang, R. G. Parr, Phys. Rev. B 37 (1988) 785.

[47] Jaguar, Jaguar 4.2. Schrödinger, Inc., Portland, OR, 1991-2000.

[48] J. Ridley, M. Zerner, Theor. Chim. Acta 32 (1973) 111.

[49] R. W. Boyd, Nonlinear Optics, Academic Press, San Diego, 1992.

[50] B. J. Orr, J. F. Ward, Mol. Phys. 20 (1971) 513.

[51] D. Fichou, B. Bachet, F. Demanze, I. Billy, G. Horowitz, F. Garnier, Adv. Mater. 8 (1996) 500. 